\title{
Development of Mycorrhiza and their Influence on Nutrient Status, Plant Growth and Innate Immunity
}

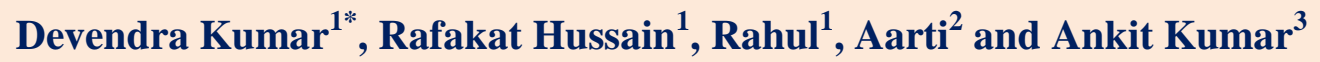 \\ ${ }^{1}$ Department of Plant Pathology, Sher-E-Kashmir University of Agricultural Sciences and \\ Technology, Jammu- Chatha 180009, India \\ ${ }^{2}$ Department of plant protection, Aligarh Muslim University Aligarh, \\ Uttar Pradesh 202001, India \\ ${ }^{3}$ Department of plant protection, Choudhary Charan Singh University Meerut, \\ Uttar Pradesh 250004, India \\ *Corresponding author
}

\section{A B S T R A C T}

\section{Keywords}

Mycorrhiza, Plant growth, Nutrient uptake, Plant defence

Article Info

Accepted:

15 November 2019

Available Online:

10 December 2019
Arbuscular mycorrhizas (AM) are mutualistic associations formed between the roots of 80 per cent of terrestrial plant species. The biotrophic AM fungus receives plant carbohydrates and in return provides the plant with mineral nutrients. AM fungi influence the physicochemical properties of the soil and directly or indirectly contributes to the release of phosphate from inorganic complexes of low solubility and also improve nutrient uptake and carbon metabolism. In a pre-contact stage sometimes also referred to as pre-symbiotic stage-mutual recognition is characterized by hyphal-branching responses elicited by plant-derived strigolactones (SLs) and plant gene expression induced by diffusible fungal-signalling molecules. In response to mechanical and chemical signals emanating from fungal hyphopodia, the contacted plant cell forms an intracellular accommodation structure, the pre-penetration apparatus (PPA) that guides intracellular fungal passage into deeper cell layers (Genre et al., 2005). Later, several authors reported a higher tolerance of mycorrhizal plants to abiotic stresses such as drought, salinity, or presence of heavy metals.

\section{Introduction}

Arbuscular mycorrhizas (AM) are mutualistic associations formed between the roots of 80 per cent of terrestrial plant species and fungi from the small phylum Glomeromycota (Schubler, et al., 2001). The soil fungi, Mycorrhiza develop specialized areas, called symbiotic interfaces, to interact with the host plant. The term mycorrhiza is derived from the 
Greek words for 'fungus' and 'root'. According to both fossil discoveries and DNA sequences, the appearance of both Mycorrhiza is almost 400 million years old. Mycorrhizal fungi develop an extensive hyphal network in the soil, the aptly named wood-wide web (Helgason et al., 1998) mycorrhiza is probably the most widespread terrestrial symbiosis (Fitter, 2005) and is formed by 70-90\% of land plant species (Smith 2008) with fungi that belong to a monophyletic phylum, the Glomeromycota (Schübler, 2001). Mycorrhiza classifications reflect anatomical aspects and identify two broad categories (Smith 2008) referred to as Ectomycorrhiza (EMs) and Endomycorrhiza, depending on whether the fungus colonizes the root intercellular spaces or develops inside cells. Endomycorrhiza are further divided into orchid, ericoid and Arbuscular mycorrhiza (AMs) other hand Ectomycorrhiza occur in certain families of woody gymnosperms (e.g., Pinaceae) and angiosperms (e.g., Dipterocarpaceae, Betulaceae) and are extremely important in many temperate and boreal forests. Early studies on mycorrhiza showed improved growth and/or yield of Mycorrhizal plants, first attributed exclusively to the improved nutritional status of the plant (Linderman, 1994). This hyphal network is specialized for nutrient (predominantly phosphate) and water uptake (Finlay 2008). In return for supplying plants with nutrients and water mycorrhiza obtain carbohydrates from plant (Solaiman 1997, Bago 2003). Therefore, mycorrhizal symbiosis contributes significantly to global phosphate and carbon cycling and influences primary productivity in terrestrial ecosystems (Fitter 2005). Soil biological performance is a key parameter affecting soil health, quality and production. Evaluating such effects can be beneficial to enhance ecological and agricultural efficiency (Artursson et al., 2006). Later, several authors reported a higher tolerance of mycorrhizal plants to abiotic stresses, such as drought, salinity, or presence of heavy metals (Miransari, 2010; Smith et al., 2010).

Plant roots exude a diverse array of biologically active compounds (Bais, 2006). Estimates suggest plants can exude up to $40 \%$ of their photosynthates from roots, representing a rich source of energy for soil microbes (Lugtenberg, 1999). Root exudates typically contain sugars, amino acids, carboxylic acids, phenolics, and other secondary metabolites, which all have the capacity to influence the occurrence, physiology, and behaviour of soil organisms. Involvement of root signals in the attraction of both pathogenic and mutualistic soil organisms is not rare. For instance, root-borne isoflavones secreted by soybean can attract the endosymbiotic N-fixing bacterium Bradyrhizobium japonicum (Subramanian 2007), but can simultaneously attract the pathogenic oomycete Phytophthora sojae (Morris 1998). Research on plant-mycorrhiza interactions have mostly focussed on the physiology of nutrient for carbon exchange and plant signal-transduction pathways controlling the interaction. Comparatively little is known about the mechanisms conferring non-nutritional benefits by mycorrhiza, such as suppression of soil-borne diseases and enhancing plant resistance to pests and diseases (Cameron, 2010).

\section{Development of mycorrhizal association with plant roots}

For the interaction between plants and AMF, strigolactones have been identified as important AMF-recruiting signals. This class of terpenoid lactones, long known as germination signals for parasitic plants, stimulates hyphal branching in AMF, thereby helping the fungus to localise host roots and so facilitate infection. The recent pioneering discovery of strigolactones as novel endogenous plant hormones in diverse 
angiosperms that range from Arabidopsis thaliana to pea and rice (Umehara). Strigolactone perception by the fungus induces the so-called presymbiotic stage, which is characterized by continued hyphal growth, increased physiological activity and profuse branching of hyphae.

The root of compatible host is infected by germ-tubes arising from spores, by hyphae growing from other propagules or by external hyphae connected to active mycorrhiza. The hyphal growth from germinating spores is dependent initially upon its own nutrient supply but stimulation of further fungal growth from germinating spores is by root exudates of a compatible plant host.

There is currently much interest in the molecular identification of fungal signalling molecules that induce symbiosis- specific responses in the host root (collectively called Myc factors). This Myc factor was found to be a diffusible molecule that induced transcriptional activation of symbiosis-related genes. Whether the production of this Myc factor is stimulated by strigolactones is unclear.

The first decisive step to mark the onset of colonization is the differentiation of the tipgrowing hypha into a hyphopodium at the root surface. The prepenetration apparatus (PPA) is a subcellular structure that predetermines the path of fungal growth through the plant cell and is formed 4-5 hours after the formation of a fungal appressorium, also called a hyphopodium. It has been shown that the formation of appressorium is induced by the root exudates produced by a compatible host plant whereas root exudates from non-host such as lupin stimulate only hyphal elongation but no formation of true appressoria in $G$. mosseae (Giovannetti et al., 1993). The initial stages of root colonisation by AMF are accompanied by transient induction of selected plant defences, followed by localised suppression at later stages of the interaction (Kapulnik et al., 1996). It is plausible that the initial induction of plant immunity is based on host recognition of microbe-associated molecular patterns (MAMPs) from the AMF. Recognition of MAMPs by pattern recognition receptors elicits a series of signalling cascades resulting in enhanced production of the plant defence hormone SA and expression of MAMP-triggered immunity.

\section{Influence of Mycorrhiza on Nutrient status plant growth and defence}

Fungal hyphae explore the soil substratum, but different AM fungi seem to use different strategies to do so. The fungal hyphal network is ideally positioned to efficiently take up nutrients and water from the soil, but only a few fungal transporters that are involved in this process, including those that transport phosphate ammonium and zinc have been cloned. The plant can control the flux of sucrose directed to the root, including the fungus. Jasmonic acid has been proposed to be involved in the regulation of sink strength of AM roots (Hause, 2002). Sucrose that is delivered to the AM root is cleaved either by symbiosis-induced sucrose synthases or invertases. In vivo studies indicate that AM fungi obtain hexoses from the plant and convert them into lipids and glycogen for long-distance transport. The mycorrhiza improved phosphate uptake is the main benefit of the AM symbiosis (Javot, 2007). The extensive hyphal network of AM fungi influences the physicochemical properties of the soil and directly or indirectly contributes to the release of phosphate from inorganic complexes of low solubility. Symbiosisinduced plant phosphate transporter genes have been identified in different plant species (Karandashov and Bucher, 2005) and accumulating evidence suggests a role for at least a subset of the corresponding proteins in 
symbiotic phosphate transport (Maeda 2006). AM fungi can accelerate decomposition and directly acquire nitrogen from organic material. A fungal amino-acid transporter and an ammonium transporter that might be involved in nitrogen uptake by extraradical hyphae have been cloned. Long-distance transport to the plant probably proceeds mainly through arginine (Govindarajulu 2005). Nitrogen is released in a carbon-free form (probably ammonium) to the plant although the ammonium transporters in the symbiotic interface membranes have not yet been identified

The transient nature of MAMP-triggered immune responses during the early stages of mycorrhization suggest that AMF employ strategies similar to those of pathogenic fungi, which secrete specific effector molecules to suppress plant immunity and establish a successful infection. Additional evidence for active immune suppression by AMF came from the discovery that the calcium/calmodulin kinase DMI3, a central regulator in the symbiotic pathway, represses early-acting defence genes. Kloppholz (2011) were the first to identify an effector protein (SP7) from Rhizophagus irregularis (syn. Glomus intraradices). This secreted protein is expressed during the initial stages of contact between the mycorrhizal fungus and roots and is translocated to the plant nucleus, where it inhibits the transcription factor ERF19 to suppress plant defence and promote infection by biotrophic fungi like $R$. irregularis. AMF induce species-specific changes in defence hormones in their hosts. Some of these hormonal changes can restrict AMF colonization, whereas others function to promote biotrophic AMF infection. Apart from plant-mediated changes in root exudates chemistry, metabolic activity by the fungus itself can also alter the chemical composition of mycorrhizal root exudates. Pulse-chase labelling experiments with $\mathrm{CO}_{2}$ revealed that plant-assimilated carbon is transferred within hours to the fungus and can be traced back in specific mycorrhizosphere bacteria a few days later. Exactly which AMF-induced changes in mycorrhizal root exudates chemistry shape the bacterial composition of the mycorrhizosphere remains difficult to predict on the basis of correlative studies and in vitro chemotaxis assays, but it is likely that a combination of primary and secondary metabolites is involved. Like AMF, rhizobacteria possess MAMPs, which can trigger MAMP induced immune responses (Boller, 2009). Wellknown examples of defence-eliciting MAMPs from bacteria are rhamnolipids, the elongation factor Tu, flagellin, and cell-wall lipopolysaccharides. The spatially confined structure of the mycorrhizosphere allows rhizobacterial strains to reach exceptionally high cell densities. Under these conditions, bacterial gene expression can be controlled by small diffusible signal molecules from members of the population themselves. This auto-induction process, known as quorum sensing (QS), allows bacteria to adjust community gene expression in accordance with their environment (Lugtenberg, 2009). Many rhizosphere-colonizing bacteria, including Pseudomonas and Burkholderia strains, employ QS to control gene expression. Some QS autoinducer molecules, like N-3oxotetradecanoyl- L-homoserine lactone, can elicit resistance in Arabidopsis to Pseudomonas syringae and Golovinomyces orontii and in barley (Hordeum vulgare) to Blumeria graminis f. sp. hordei.

\section{Outlook}

Over the past few years, a novel and unexpected developmental capacity of plant cells has been discovered that is essential for the intracellular uptake of AM fungi. Plant genetics will continue to be a major tool in the identification of genes that are required for $\mathrm{AM}$ development and function. It is expected 
that in the near future, the chemical structure of the fungal Myc factor that triggers the symbiotic responses of the root will be published, which will help us to identify the cognate plant receptors. To unlock the potential of AM for sustainable agriculture, we must identify the key molecular players equally importantly, we must investigate the natural variation for AM function and responsiveness within biodiversity collections of important crop plants120 and between different fungal lineages. The long-term aim is to identify or design crop-fungus combinations with optimized AM performance, which would be instrumental in reducing the application of fertilizer and energy input, a goal that is mandatory in a world of depleting non-renewable resources121 Mycorrhizal fungi positively influence the concentration of photosynthetic pigmentation, maintain ionic balance increase accumulation of antioxidants and osmoprotectants. In addition to improved plant nutrient uptake, mycorrhizal fungi play a significant role in alleviation of salt, drought and heavy metal stresses and plant protection against disease. In the future, mycorrhizosphere management must become one of the viable and ecosystem friendly solutions to managing plant diseases.

\section{References}

Akiyama, K., Matsuzaki, K. and Hayashi, H. 2005. Plant sesquiterpenes induce hyphal branching in Arbuscular mycorrhizal fungi. Nature, 435: 82427.

Artursson V., Finlay R. D. and Jansson J. K. 2006. Interactions between arbuscular mycorrhizal fungi and bacteria and their potential for stimulating plant growth. Environ Microbiol., 8: 1-10

Bago B. 2003. Carbon export from arbuscular mycorrhizal roots involves the translocation of carbohydrate as well as lipid. Plant Physiol., 131: 14961507

Bais H. P. 2006. The role of root exudates in rhizosphere interactions with plants and other organisms. Annu. Rev. Plant Biol., 57: 233-266.

Boller T. and Felix G. 2009. A renaissance of elicitors: perception of microbeassociated molecular patterns and danger signals by pattern-recognition receptors. Annu. Rev. Plant Biol., 60: 379-406.

Cameron D. 2010. Arbuscular mycorrhizal fungi as agro-ecosystem engineers. Plant Soil., 333: 1-5.

Finlay R. D. 2008. Ecological aspects of mycorrhizal symbiosis: with special emphasis on the functional diversity of interactions involving the extraradical mycelium. J. Exp. Bot., 59: 1115-1126

Fitter A. H. 2005. Darkness visible: reflections on underground ecology. J. Ecol., 93: 231-243

Genre, A., Chabaud, M., Timmers, T., Bonfante, P. and Barker, DG. 2005. Arbuscular mycorrhizal fungi elicit a novel intracellular apparatus in Medicago truncatula root epidermal cells before infection. Plant Cell 17:3489-99.

Giovannetti M., Avio L., Sabrana C. and Citernesi A. S. 1993. Factors affecting appressorium development in the VAM fungus Glomus mosseae (Nicol and Gerd) Gerd and Trappe. New Phytol., 123: 114- 122.

Govindarajulu, M. 2005. Nitrogen transfer in the arbuscular mycorrhizal symbiosis. Nature 435, 819-823.

Hause, B., Maier, W., Miersch, O., Kramell, R. and Strack, D. 2002. Induction of jasmonate biosynthesis in arbuscular mycorrhizal barley roots. Plant Physiol. 130, 1213-1220.

Helgason T., Daniell T. J., Husband R., Fitter A. H. and Young J. P. W. 1998. 
Ploughing up the wood-wide web, Nature, 394: 431

Javot, H., Pumplin, N. and Harrison, M. J. 2007. Phosphate in the arbuscular mycorrhizal symbiosis: transport properties and regulatory roles. Plant Cell Environ. 30, 310-322.

Kapulnik Y. 1996. Suppression of defence responses in mycorrhizal alfalfa and tobacco roots. New Phytol., 133: 5964

Karandashov, V. and Bucher, M. 2005. Symbiotic phosphate transport in arbuscular mycorrhizas. Trends Plant Sci. 10, 22-29.

Kloppholz S. 2011. A secreted fungal effector of Glomus intraradices promotes symbiotic biotrophy. Curr. Biol., 21: 1204-1209

Linderman R. G. 1994. Role of VAM fungi in biocontrol, pp. 1-26, in F. L. Pfleger and R. G. Linderman (eds.), Mycorrhizae and Plant Health. APS Press, St. Paul, MN.

Lugtenberg B. and Kamilova F. 2009. Plantgrowth-promoting rhizobacteria. Annu. Rev. Microbiol., 63: 541-556

Lugtenberg B.J. 1999. Tomato seed and root exudate sugars: composition, utilization by Pseudomonas biocontrol strains and role in rhizosphere colonization. Environ. Microbiol., 1: 439-446

Maeda, D. 2006. Knockdown of an Arbuscular mycorrhiza-inducible phosphate transporter gene of Lotus japonicus suppresses mutualistic symbiosis. Plant Cell Physiol. 47, 807817.

Miransari M. 2010. Contribution of arbuscular mycorrhizal symbiosis to plant growth under different types of soil stress. Plant Biol., 12: 563-569.

Morris P. F. 1998. Chemotropic and contact responses of Phytophthora sojae hyphae to soybean isoflavonoids and artificial substrates. Plant Physiol., 117:1171-1178.

Schußler A, Schwarzott D, and Walker C. 2001. A new fungal phylum, the Glomeromycota, phylogeny and evolution. Mycol Res., 105: 14131421.

Smith S. E. and Read D. J. 2008. Mycorrhizal Symbiosis. 3rd ed. Academic Press; Linderman RG. Mycorrhizal Interactions with the rhizosphere microflora-the Mycorrhizosphere effect. Phytopathology; 78: 366-371.

Smith S., Facelli E., Pope S. and Andrew Smith F. 2010. Plant performance in stressful environments: interpreting new and established knowledge of the roles of arbuscular mycorrhizas. Plant Soil, 326: 3-2

Solaiman M. D. Z. and Saito M. 1997. Use of sugars by intraradical hyphae of arbuscular mycorrhizal fungi revealed by radiorespirometry. New Phytol., 136: 533-538

Subramanian S., et al., 2007. Distinct, crucial roles of flavonoids during legume nodulation. Trends Plant Sci., 12: 282285.

\section{How to cite this article:}

Devendra Kumar, Rafakat Hussain, Rahul, Aarti and Ankit Kumar. 2019. Development of Mycorrhiza and their Influence on Nutrient Status, Plant Growth and Innate Immunity. Int.J.Curr.Microbiol.App.Sci. 8(12): 1886-1891. doi: https://doi.org/10.20546/ijcmas.2019.812.225 